\title{
Inequalities of Convex Functions and Self-Adjoint Operators
}

\author{
Zlatko Pavić \\ Mechanical Engineering Faculty in Slavonski Brod, University of Osijek, Trg Ivane Brlić Mažuranić 2, 35000 Slavonski Brod, Croatia \\ Correspondence should be addressed to Zlatko Pavić; zpavic@sfsb.hr \\ Received 28 November 2013; Accepted 4 January 2014; Published 9 February 2014 \\ Academic Editor: Palle E. Jorgensen \\ Copyright (C) 2014 Zlatko Pavić. This is an open access article distributed under the Creative Commons Attribution License, which \\ permits unrestricted use, distribution, and reproduction in any medium, provided the original work is properly cited. \\ The paper offers generalizations of the Jensen-Mercer inequality for self-adjoint operators and generally convex functions. The \\ obtained results are applied to define the quasi-arithmetic operator means without using operator convexity. The version of the \\ harmonic-geometric-arithmetic operator mean inequality is derived as an example.
}

\section{Introduction}

Throughout the paper we will use a real interval $\mathscr{I}$ with the nonempty interior and real segments $[a, b]$ and $(a, b)$ with $a<$ $b$.

We briefly summarize a development path of the operator form of Jensen's inequality. Let $\mathscr{H}$ and $\mathscr{K}$ be Hilbert spaces, let $\mathrm{B}(\mathscr{H})$ and $\mathrm{B}(\mathscr{K})$ be associated $\mathrm{C}^{*}$-algebras of bounded linear operators, and let $1_{H}$ and $1_{K}$ be their identity operators.

Combining the results from $[1,2]$, it follows that every operator convex function $f: \mathscr{I} \rightarrow \mathbb{R}$ satisfies the Schwarz inequality

$$
f(\Phi(A)) \leq \Phi(f(A)),
$$

where $\Phi: \mathrm{B}(\mathscr{H}) \rightarrow \mathrm{B}(\mathscr{K})$ is a positive linear mapping such that $\Phi\left(1_{H}\right)=1_{K}$ and $A \in \mathrm{B}(\mathscr{H})$ is a self-adjoint operator with the spectrum $\operatorname{Sp}(A) \subseteq \mathscr{I}$. The above inequality was extended in [3] to the inequality

$$
f\left(\sum_{i=1}^{n} \Phi_{i}\left(A_{i}\right)\right) \leq \sum_{i=1}^{n} \Phi_{i}\left(f\left(A_{i}\right)\right),
$$

where $\Phi_{i}: \mathrm{B}(\mathscr{H}) \rightarrow \mathrm{B}(\mathscr{K})$ are positive linear mappings such that $\sum_{i=1}^{n} \Phi_{i}\left(1_{H}\right)=1_{K}$ and $A_{i} \in \mathrm{B}(\mathscr{H})$ are self-adjoint operators with spectra $\operatorname{Sp}\left(A_{i}\right) \subseteq \mathscr{I}$. The operator inequality of (2) was formulated for convex (without operator) continuous functions in [4] assuming the spectral conditions: $\operatorname{Sp}(A) \subseteq[a, b]$ and $\operatorname{Sp}\left(A_{i}\right) \cap(a, b)=\emptyset$ for all $A_{i}$, where $A=\sum_{i=1}^{n} \Phi_{i}\left(A_{i}\right)$.
Including positive operators $P_{i} \in \mathrm{B}(\mathscr{H})$ satisfying $\sum_{i=1}^{n} \Phi_{i}\left(P_{i}\right)=1_{K}$, we have that every convex continuous function $f: \mathscr{I} \rightarrow \mathbb{R}$ satisfies the inequality

$$
f\left(\sum_{i=1}^{n} \Phi_{i}\left(P_{i}^{1 / 2} A_{i} P_{i}^{1 / 2}\right)\right) \leq \sum_{i=1}^{n} \Phi_{i}\left(P_{i}^{1 / 2} f\left(A_{i}\right) P_{i}^{1 / 2}\right)
$$

if provided the spectral conditions: $\operatorname{Sp}(A) \subseteq[a, b]$ and $\operatorname{Sp}\left(A_{i}\right) \cap(a, b)=\emptyset$ for all self-adjoint operators $A_{i}$, and the operator sum $A=\sum_{i=1}^{n} \Phi_{i}\left(P_{i}^{1 / 2} A_{i} P_{i}^{1 / 2}\right)$. The inequality in (3) is possible because the operators $P_{i}^{1 / 2} A_{i} P_{i}^{1 / 2}$ and $P_{i}^{1 / 2} f\left(A_{i}\right) P_{i}^{1 / 2}$ are self-adjoint.

\section{Discrete and Operator Inequalities for Convex Functions and Trinomial Affine Combinations}

2.1. Discrete Variants. Every number $x \in \mathbb{R}$ can be uniquely presented as the binomial affine combination

$$
x=\frac{b-x}{b-a} a+\frac{x-a}{b-a} b
$$

which is convex if and only if the number $x$ belongs to the interval $[a, b]$. Given the function $f: \mathbb{R} \rightarrow \mathbb{R}$, let $f_{\{a, b\}}^{\text {line }}$ : $\mathbb{R} \rightarrow \mathbb{R}$ be the function of the chord line passing through the points $A(a, f(a))$ and $B(b, f(b))$ of the graph of $f$. Applying the affinity of $f_{\{a, b\}}^{\text {line }}$ to the combination in (4), we get

$$
f_{\{a, b\}}^{\text {line }}(x)=\frac{b-x}{b-a} f(a)+\frac{x-a}{b-a} f(b) .
$$


If the function $f$ is convex, then we have the inequality

$$
f(x) \leq f_{\{a, b\}}^{\text {line }}(x) \quad \text { if } x \in[a, b],
$$

and the reverse inequality

$$
f(x) \geq f_{\{a, b\}}^{\text {line }}(x) \quad \text { if } x \notin(a, b) .
$$

Let $\alpha, \beta, \gamma \in \mathbb{R}$ be coefficients such that $\alpha+\beta-\gamma=1$. Let $a, b, c \in \mathbb{R}$ be points where $a<b$. We consider the affine combination $\alpha a+\beta b-\gamma c$. Inserting the affine combination $c=\lambda a+\mu b$ assuming that $\lambda+\mu=1$, we get the binomial form

$$
\alpha a+\beta b-\gamma c=(\alpha-\gamma \lambda) a+(\beta-\gamma \mu) b .
$$

Lemma 1. Let $\alpha, \beta, \gamma \in[0,1]$ be coefficients such that $\alpha+\beta-$ $\gamma=1$. Let $a, b, c \in \mathbb{R}$ be points such that $a<b$ and $c \in[a, b]$.

Then the affine combination

$$
\alpha a+\beta b-\gamma c \in[a, b]
$$

and every convex function $f:[a, b] \rightarrow \mathbb{R}$ satisfies the inequality

$$
f(\alpha a+\beta b-\gamma c) \leq \alpha f(a)+\beta f(b)-\gamma f(c) .
$$

Proof. The condition $c=\lambda a+\mu b \in[a, b]$ involves $\lambda, \mu \in$ $[0,1]$. Then the binomial combination of the right-hand side in (8) is convex since its coefficients $\alpha-\gamma \lambda \geq \alpha-\gamma=1-\beta \geq 0$ and also $\beta-\gamma \mu \geq 0$. So, the combination $\alpha a+\beta b-\gamma$ belongs to $[a, b]$. Applying the inequality in (6) and the affinity of $f_{\{a, b\}}^{\text {line }}$, we get

$$
\begin{aligned}
f(\alpha a+\beta b-\gamma c) & \leq f_{\{a, b\}}^{\text {line }}(\alpha a+\beta b-\gamma c) \\
& =\alpha f(a)+\beta f(b)-\gamma f_{\{a, b\}}^{\text {line }}(c) \\
& \leq \alpha f(a)+\beta f(b)-\gamma f(c)
\end{aligned}
$$

because $f_{\{a, b\}}^{\text {line }}(c) \geq f(c)$.

Lemma 1 is trivially true if $a=b$. It is also valid for $\gamma \in[-1,1]$ because then the observed affine combinations with $\gamma \leq 0$ become convex, and associated inequalities follow from Jensen's inequality. The similar combinations including $\gamma \in[-1,1]$ were observed in [5, Corollary 11 and Theorem 12] additionally using a monotone function $g$. If $\alpha=\beta=\gamma=$ 1 , then the inequality in (10) is reduced to simple Mercer's variant of Jensen's inequality obtained in [6].

Lemma 2. Let $\alpha, \beta, \gamma \in[1, \infty)$ be coefficients such that $\alpha+\beta-$ $\gamma=1$. Let $a, b, c \in \mathbb{R}$ be points such that $a<b$ and $c \notin(a, b)$.

Then the affine combination

$$
\alpha a+\beta b-\gamma c \notin(a, b)
$$

and every convex function $f: \mathscr{I} \rightarrow \mathbb{R}$, where $\mathscr{I}=$ $\operatorname{conv}\{a, b, c\}$ satisfies the inequality

$$
f(\alpha a+\beta b-\gamma c) \geq \alpha f(a)+\beta f(b)-\gamma f(c) .
$$

Proof. The condition $c=\lambda a+\mu b \notin(a, b)$ entails $\lambda \leq 0$ or $\lambda \geq 1$, and the coefficients of the binomial form of (8) satisfy $\alpha-\gamma \lambda \geq \alpha \geq 1$ if $\lambda \leq 0$, or $\alpha-\gamma \lambda \leq \alpha-\gamma=1-\beta \leq 0$ if $\lambda \geq 1$. So, the combination $\alpha a+\beta b-\gamma$ does not belong to $(a, b)$. Applying the inequality in (7), we get the series of inequalities as in (11) but with the reverse inequality signs.

It is not necessary to require $\gamma \in[1, \infty)$ in Lemma 2, because it follows from the other coefficient conditions.

2.2. Operator Variants. We write $A \leq B$ for self-adjoint operators $A, B \in \mathscr{B}(\mathscr{H})$ if the inner product inequality $\langle A x, x\rangle \leq\langle B x, x\rangle$ holds for every vector $x \in \mathscr{H}$. A selfadjoint operator $A$ is positive (nonnegative) if it is greater than or equal to null operator $(A \geq 0)$. If $\operatorname{Sp}(A) \subseteq \mathscr{I}$ and $f, g$ : $\mathscr{I} \rightarrow \mathbb{R}$ are continuous functions such that $f(x) \leq g(x)$ for every $x \in \operatorname{Sp}(A)$, then the operator inequality $f(A) \leq g(A)$ is valid. The bounds of a self-adjoint operator $A$ are defined with

$$
a_{A}=\inf _{\|x\|=1}\langle A x, x\rangle, \quad b_{A}=\sup _{\|x\|=1}\langle A x, x\rangle,
$$

and its spectrum $\operatorname{Sp}(A)$ is contained in $\left[a_{A}, b_{A}\right]$ wherein we have the operator inequality

$$
a_{A} 1_{H} \leq A \leq b_{A} 1_{H}
$$

More details on the theory of bounded operators and their inequalities can be found in [7]. The operator versions of Lemmas 1 and 2 follow.

Corollary 3. Let $\alpha, \beta, \gamma \in[0,1]$ be coefficients such that $\alpha+$ $\beta-\gamma=1$. Let $A \in \mathrm{B}(\mathscr{H})$ be a self-adjoint operator such that $\operatorname{Sp}(A) \subseteq[a, b]$.

Then

$$
S p\left(\alpha a 1_{H}+\beta b 1_{H}-\gamma A\right) \subseteq[a, b],
$$

and every convex continuous function $f:[a, b] \rightarrow \mathbb{R}$ satisfies the inequality

$$
f\left(\alpha a 1_{H}+\beta b 1_{H}-\gamma A\right) \leq \alpha f(a) 1_{H}+\beta f(b) 1_{H}-\gamma f(A) .
$$

Proof. The spectral inclusion in (16) follows from the inclusion in (9). Using the affinity of the function $f_{\{a, b\}}^{\text {line }}$ and the operator inequalities $f_{\{a, b\}}^{\text {line }}(\cdot) \geq f(\cdot)$, we can replace the discrete inequalities in (11) with the operator inequalities.

Corollary 4. Let $\alpha, \beta, \gamma \in[1, \infty)$ be coefficients such that $\alpha+$ $\beta-\gamma=1$. Let $A \in \mathrm{B}(\mathscr{H})$ be a self-adjoint operator such that $\operatorname{Sp}(A) \cap(a, b)=\emptyset$.

Then

$$
S p\left(\alpha a 1_{H}+\beta b 1_{H}-\gamma A\right) \cap(a, b)=\emptyset,
$$

and every convex continuous function $f: \mathscr{I} \rightarrow \mathbb{R}$, where $\mathscr{I}$ contains $\operatorname{Sp}(A)$ and $[a, b]$, satisfies the inequality

$$
f\left(\alpha a 1_{H}+\beta b 1_{H}-\gamma A\right) \geq \alpha f(a) 1_{H}+\beta f(b) 1_{H}-\gamma f(A) .
$$




\section{Main Results}

We want to extend and generalize the inequalities in (17) and (19) including positive operators and positive linear mappings. The main results are Theorems 8 and 9.

Lemma 5. Let $\Phi_{i}: \mathrm{B}(\mathscr{H}) \rightarrow \mathrm{B}(\mathscr{K})$ be linear mappings and let $P_{i} \in \mathrm{B}(\mathscr{H})$ be positive linear operators so that $\sum_{i=1}^{n} \Phi_{i}\left(P_{i}\right)=$ $1_{K}$. Let $A_{i} \in \mathrm{B}(\mathscr{H})$ be self-adjoint operators.

Then every affine function $g(x)=u x+v$, where $u$ and $v$ are real constants, satisfies the operator equality

$$
g\left(\sum_{i=1}^{n} \Phi_{i}\left(P_{i}^{1 / 2} A_{i} P_{i}^{1 / 2}\right)\right)=\sum_{i=1}^{n} \Phi_{i}\left(P_{i}^{1 / 2} g\left(A_{i}\right) P_{i}^{1 / 2}\right) .
$$

Proof. Applying the affinity of the function $g$ and the assumption $\sum_{i=1}^{n} \Phi_{i}\left(P_{i}\right)=1_{K}$, it follows that

$$
\begin{aligned}
g\left(\sum_{i=1}^{n} \Phi_{i}\left(P_{i}^{1 / 2} A_{i} P_{i}^{1 / 2}\right)\right) & =u \sum_{i=1}^{n} \Phi_{i}\left(P_{i}^{1 / 2} A_{i} P_{i}^{1 / 2}\right)+v 1_{K} \\
& =u \sum_{i=1}^{n} \Phi_{i}\left(P_{i} A_{i}\right)+v \sum_{i=1}^{n} \Phi_{i}\left(P_{i}\right) \\
& =\sum_{i=1}^{n} \Phi_{i}\left(P_{i}^{1 / 2}\left(u A_{i}+v 1_{H}\right) P_{i}^{1 / 2}\right) \\
& =\sum_{i=1}^{n} \Phi_{i}\left(P_{i}^{1 / 2} g\left(A_{i}\right) P_{i}^{1 / 2}\right)
\end{aligned}
$$

achieving the equality in (20).

Lemma 6. Let $\Phi_{i}: \mathrm{B}(\mathscr{H}) \rightarrow \mathrm{B}(\mathscr{K})$ be positive linear mappings and let $P_{i} \in \mathrm{B}(\mathscr{H})$ be positive linear operators so that $\sum_{i=1}^{n} \Phi_{i}\left(P_{i}\right)=1_{K}$. Let $A_{i} \in \mathrm{B}(\mathscr{H})$ be self-adjoint operators such that $\operatorname{Sp}\left(A_{i}\right) \subseteq[a, b]$.

Then the spectrum of the operator sum $A=$ $\sum_{i=1}^{n} \Phi_{i}\left(P_{i}^{1 / 2} A_{i} P_{i}^{1 / 2}\right)$ is contained in $[a, b]$.

Proof. Applying the positive operators $P_{i}$ and the positive mappings $\Phi_{i}$ to the assumed spectral inequalities

$$
a 1_{H} \leq A_{i} \leq b 1_{H},
$$

we get

$$
a \Phi_{i}\left(P_{i}\right) \leq \Phi_{i}\left(P_{i}^{1 / 2} A_{i} P_{i}^{1 / 2}\right) \leq b \Phi_{i}\left(P_{i}\right) .
$$

Summing the above inequalities and using the assumption $\sum_{i=1}^{n} \Phi_{i}\left(P_{i}\right)=1_{K}$, we have

$$
a 1_{K} \leq \sum_{i=1}^{n} \Phi_{i}\left(P_{i}^{1 / 2} A_{i} P_{i}^{1 / 2}\right) \leq b 1_{K}
$$

which provides that $\operatorname{Sp}(A) \subseteq[a, b]$.

Corollary 7. Let $\Phi_{i}: \mathrm{B}(\mathscr{H}) \rightarrow \mathrm{B}(\mathscr{K})$ be positive linear mappings and let $P_{i} \in \mathrm{B}(\mathscr{H})$ be positive linear operators so that $\sum_{i=1}^{n} \Phi_{i}\left(P_{i}\right)=1_{K}$. Let $A_{i} \in \mathrm{B}(\mathscr{H})$ be self-adjoint operators such that $\operatorname{Sp}\left(A_{i}\right) \subseteq[a, b]$.

Then every convex continuous function $f:[a, b] \rightarrow \mathbb{R}$ satisfies the inequality

$$
\begin{aligned}
& \max \left\{f\left(\sum_{i=1}^{n} \Phi_{i}\left(P_{i}^{1 / 2} A_{i} P_{i}^{1 / 2}\right)\right), \sum_{i=1}^{n} \Phi_{i}\left(P_{i}^{1 / 2} f\left(A_{i}\right) P_{i}^{1 / 2}\right)\right\} \\
& \quad \leq f_{\{a, b\}}^{\text {line }}\left(\sum_{i=1}^{n} \Phi_{i}\left(P_{i}^{1 / 2} A_{i} P_{i}^{1 / 2}\right)\right) \\
& \quad \leq \max \left\{f(a) 1_{K}, f(b) 1_{K}\right\} .
\end{aligned}
$$

Proof. The inequality in (25) is the consequence of Lemmas 5 and 6 , and the discrete inequality

$$
\begin{aligned}
& \max \left\{f\left(\sum_{i=1}^{n} p_{i} x_{i}\right), \sum_{i=1}^{n} p_{i} f\left(x_{i}\right)\right\} \\
& \leq f_{\{a, b\}}^{\text {line }}\left(\sum_{i=1}^{n} p_{i} x_{i}\right) \\
& \leq \max \{f(a), f(b)\},
\end{aligned}
$$

where $x_{i} \in[a, b]$ are points and $p_{i} \in[0,1]$ are coefficients of the sum equal to 1 .

Theorem 8. Let $\alpha, \beta, \gamma \in[0,1]$ be coefficients such that $\alpha+$ $\beta-\gamma=1$. Let $\Phi_{i}: \mathrm{B}(\mathscr{H}) \rightarrow \mathrm{B}(\mathscr{K})$ be positive linear mappings and let $P_{i} \in \mathrm{B}(\mathscr{H})$ be positive linear operators so that $\sum_{i=1}^{n} \Phi_{i}\left(P_{i}\right)=1_{K}$. Let $A_{i} \in \mathrm{B}(\mathscr{H})$ be self-adjoint operators such that $\operatorname{Sp}\left(A_{i}\right) \subseteq[a, b]$.

Then the spectrum of the operator

$$
\bar{A}=\alpha a 1_{K}+\beta b 1_{K}-\gamma \sum_{i=1}^{n} \Phi_{i}\left(P_{i}^{1 / 2} A_{i} P_{i}^{1 / 2}\right)
$$

is contained in $[a, b]$, and every convex continuous function $f$ : $[a, b] \rightarrow \mathbb{R}$ satisfies the inequality

$$
f(\bar{A}) \leq \alpha f(a) 1_{K}+\beta f(b) 1_{K}-\gamma \sum_{i=1}^{n} \Phi_{i}\left(P_{i}^{1 / 2} f\left(A_{i}\right) P_{i}^{1 / 2}\right) .
$$

If the function $f$ is concave, then the reverse inequality is valid in (28).

Proof. Taking the operator sum $A=\sum_{i=1}^{n} \Phi_{i}\left(P_{i}^{1 / 2} A_{i} P_{i}^{1 / 2}\right)$, the spectral inclusion $\operatorname{Sp}(\bar{A}) \subseteq[a, b]$ follows from Lemma 6 and 
the inclusion in (16). Assuming and applying the convexity of $f$ and the affinity of $f_{\{a, b\}}^{\text {line }}$ according to Lemma 5, we get

$$
\begin{aligned}
f(\bar{A}) \leq & f_{\{a, b\}}^{\text {line }}\left(\alpha a 1_{K}+\beta b 1_{K}-\gamma \sum_{i=1}^{n} \Phi_{i}\left(P_{i}^{1 / 2} A_{i} P_{i}^{1 / 2}\right)\right) \\
= & \alpha f(a) 1_{K}+\beta f(b) 1_{K} \\
& -\gamma f_{\{a, b\}}^{\text {line }}\left(\sum_{i=1}^{n} \Phi_{i}\left(P_{i}^{1 / 2} A_{i} P_{i}^{1 / 2}\right)\right) \\
= & \alpha f(a) 1_{K}+\beta f(b) 1_{K}-\gamma \sum_{i=1}^{n} \Phi_{i}\left(P_{i}^{1 / 2} f_{\{a, b\}}^{\text {line }}\left(A_{i}\right) P_{i}^{1 / 2}\right) \\
\leq & \alpha f(a) 1_{K}+\beta f(b) 1_{K}-\gamma \sum_{i=1}^{n} \Phi_{i}\left(P_{i}^{1 / 2} f\left(A_{i}\right) P_{i}^{1 / 2}\right)
\end{aligned}
$$

because $f_{\{a, b\}}^{\text {line }}\left(A_{i}\right) \geq f\left(A_{i}\right)$.

The version of Theorem 8 for $\alpha=\beta=\gamma=1$ and all $P_{i}=1_{H}$ was obtained in [8] as the main result.

Theorem 9. Let $\alpha, \beta, \gamma \in[1, \infty)$ be coefficients such that $\alpha+\beta-\gamma=1$. Let $\Phi_{i}: \mathrm{B}(\mathscr{H}) \rightarrow \mathrm{B}(\mathscr{K})$ be positive linear mappings and let $P_{i} \in \mathrm{B}(\mathscr{H})$ be positive linear operators so that $\sum_{i=1}^{n} \Phi_{i}\left(P_{i}\right)=1_{K}$. Let $A_{i} \in \mathrm{B}(\mathscr{H})$ be self-adjoint operators such that $\operatorname{Sp}\left(A_{i}\right) \cap(a, b)=\emptyset$, and let $A=\sum_{i=1}^{n} \Phi_{i}\left(P_{i}^{1 / 2} A_{i} P_{i}^{1 / 2}\right)$ be the operator sum such that $\operatorname{Sp}(A) \cap(a, b)=\emptyset$.

Then the spectrum of the operator

$$
\bar{A}=\alpha a 1_{K}+\beta b 1_{K}-\gamma \sum_{i=1}^{n} \Phi_{i}\left(P_{i}^{1 / 2} A_{i} P_{i}^{1 / 2}\right)
$$

satisfies the relation $\operatorname{Sp}(\bar{A}) \cap(a, b)=\emptyset$, and every convex continuous function $f: \mathscr{I} \rightarrow \mathbb{R}$, where $\mathscr{I}$ contains all spectra and $[a, b]$, satisfies the inequality

$$
\begin{aligned}
f(\bar{A}) \geq & \alpha f(a) 1_{K}+\beta f(b) 1_{K} \\
& -\gamma \sum_{i=1}^{n} \Phi_{i}\left(P_{i}^{1 / 2} f\left(A_{i}\right) P_{i}^{1 / 2}\right) .
\end{aligned}
$$

If the function $f$ is concave, then the reverse inequality is valid in (31).

Proof. The relation $\operatorname{Sp}(\bar{A}) \cap(a, b)=\emptyset$ is the consequence of the relation in (18). Assuming and using the convexity of $f$ and the affinity of $f_{\{a, b\}}^{\text {line }}$, as well as the inequalities $f_{\{a, b\}}^{\text {line }}\left(A_{i}\right) \leq$ $f\left(A_{i}\right)$, we get the series of inequalities as in (29) but with the reverse inequality signs.

\section{Application to Quasi-Arithmetic Means}

In applications of convexity to quasi-arithmetic means, we use strictly monotone continuous functions $\varphi, \psi: \mathscr{I} \rightarrow \mathbb{R}$ such that the function $\psi \circ \varphi^{-1}$ is convex, in which case we say that $\psi$ is $\varphi$-convex. A similar notation is used for concavity. This terminology is taken from [9, Definition 1.19].

A continuous function $f: \mathscr{I} \rightarrow \mathbb{R}$ is said to be operator increasing on $\mathscr{I}$ if $A \leq B$ implies $f(A) \leq f(B)$ for every pair of self-adjoint operators $A, B \in \mathrm{B}(\mathscr{H})$ with spectra in $\mathscr{I}$. A function $f$ is said to be operator decreasing if the function $-f$ is operator increasing.

Take an operator affine combination

$$
\bar{A}=\alpha a 1_{K}+\beta b 1_{K}-\gamma \sum_{i=1}^{n} \Phi_{i}\left(P_{i}^{1 / 2} A_{i} P_{i}^{1 / 2}\right)
$$

as in Theorem 8 . If $\varphi:[a, b] \rightarrow \mathbb{R}$ is a strictly monotone continuous function, we define the $\varphi$-quasi-arithmetic mean of the combination $\bar{A}$ as the operator

$$
\begin{aligned}
M_{\varphi}(\bar{A})=\varphi^{-1}\left(\alpha \varphi(a) 1_{K}+\beta \varphi(b) 1_{K}\right. \\
\left.\quad-\gamma \sum_{i=1}^{n} \Phi_{i}\left(P_{i}^{1 / 2} \varphi\left(A_{i}\right) P_{i}^{1 / 2}\right)\right) .
\end{aligned}
$$

The spectrum of the operator $M_{\varphi}(\bar{A})$ is contained in $[a, b]$ because the spectrum of the operator

$$
\bar{A}_{\varphi}=\alpha \varphi(a) 1_{K}+\beta \varphi(b) 1_{K}-\gamma \sum_{i=1}^{n} \Phi_{i}\left(P_{i}^{1 / 2} \varphi\left(A_{i}\right) P_{i}^{1 / 2}\right)
$$

is contained in $\varphi([a, b])$. The quasi-arithmetic means defined in (33) are invariant with respect to the affinity; that is, the equality

$$
M_{u \varphi+v}(\bar{A})=M_{\varphi}(\bar{A})
$$

holds for all pairs of real numbers $u \neq 0$ and $v$. Indeed, if $\psi(x)=u \varphi(x)+v$, then

$$
\begin{gathered}
\bar{A}_{\psi}=u \bar{A}_{\varphi}+v 1_{K}, \\
\psi^{-1}(x)=\varphi^{-1}\left(\frac{1}{u}(x-v)\right),
\end{gathered}
$$

and therefore, it follows that

$$
\begin{aligned}
M_{\psi}(\bar{A}) & =\psi^{-1}\left(\bar{A}_{\psi}\right)=\varphi^{-1}\left(\frac{1}{u}\left(\bar{A}_{\psi}-v 1_{K}\right)\right) \\
& =\varphi^{-1}\left(\bar{A}_{\varphi}\right)=M_{\varphi}(\bar{A}) .
\end{aligned}
$$

The order of the pair of quasi-arithmetic means $M_{\varphi}$ and $M_{\psi}$ depends on convexity of the function $\psi \circ \varphi^{-1}$ and monotonicity of the function $\psi$. Theorem 8 can be applied to operator means as follows.

Corollary 10. Let $\bar{A}$ be an affine combination as in (32) satisfying the assumptions of Theorem 8 . Let $\varphi, \psi:[a, b] \rightarrow \mathbb{R}$ be strictly monotone continuous functions. 
If $\psi$ is either $\varphi$-convex with operator increasing $\psi^{-1}$ or $\varphi$-concave with operator decreasing $\psi^{-1}$, then one has the inequality

$$
M_{\varphi}(\bar{A}) \leq M_{\psi}(\bar{A}) .
$$

If $\psi$ is either $\varphi$-convex with operator decreasing $\psi^{-1}$ or $\varphi$ concave with operator increasing $\psi^{-1}$, then one has the reverse inequality in (38).

Proof. Let us prove the case in which $\psi$ is $\varphi$-convex with operator increasing $\psi^{-1}$. Put $[c, d]=\varphi([a, b])$. Applying the inequality in (28) of Theorem 8 to the affine combination $\bar{A}_{\varphi}$ of (34) with $\operatorname{Sp}\left(\bar{A}_{\varphi}\right) \subseteq[c, d]$ and the convex function $f=\psi \circ \varphi^{-1}:[c, d] \rightarrow \mathbb{R}$, we get

$$
\psi \circ \varphi^{-1}\left(\bar{A}_{\varphi}\right) \leq \bar{A}_{\psi} .
$$

Assigning the increasing function $\psi^{-1}$ to the above inequality, we attain

$$
M_{\varphi}(\bar{A})=\varphi^{-1}\left(\bar{A}_{\varphi}\right) \leq \psi^{-1}\left(\bar{A}_{\psi}\right)=M_{\psi}(\bar{A})
$$

which finishes the proof.

Using Corollary 10 we get the following version of the harmonic-geometric-arithmetic mean inequality for operators.

Corollary 11. If $\bar{A}$ is an affine operator combination as in (32) satisfying the assumptions of Theorem 8 with the addition that $[a, b] \subset(0, \infty)$, then one has the harmonic-geometricarithmetic operator inequality

$$
\begin{aligned}
& \left(\left(\frac{\alpha}{a}+\frac{\beta}{b}\right) 1_{K}-\gamma \sum_{i=1}^{n} \Phi_{i}\left(P_{i}^{1 / 2} A_{i}^{-1} P_{i}^{1 / 2}\right)\right)^{-1} \\
& \quad \leq \ln a^{\alpha} b^{\beta} 1_{K}-\gamma \exp \left(\sum_{i=1}^{n} \Phi_{i}\left(P_{i}^{1 / 2}\left(\ln A_{i}\right) P_{i}^{1 / 2}\right)\right) \\
& \leq(\alpha a+\beta b) 1_{K}-\gamma \sum_{i=1}^{n} \Phi_{i}\left(P_{i}^{1 / 2} A_{i} P_{i}^{1 / 2}\right) .
\end{aligned}
$$

Proof. To prove the left-hand side of the inequality in (41) we use the functions $\varphi(x)=\ln x$ and $\psi(x)=x^{-1}$. Then $\psi \circ \varphi^{-1}(x)=\exp (-x)$ and $\psi^{-1}(x)=x^{-1}$, so $\psi$ is $\varphi$-convex and $\psi^{-1}(x)=x^{-1}$ is operator decreasing. Applying Corollary 10 to this case, we have

$$
M_{\ln x}(\bar{A}) \geq M_{x^{-1}}(\bar{A}) .
$$

To prove the right-hand side we use the functions $\varphi(x)=$ $\ln x$ and $\psi(x)=x$. Then $\psi \circ \varphi^{-1}(x)=\exp x$ and $\psi^{-1}(x)=x$, so $\psi$ is $\varphi$-convex and $\psi^{-1}(x)=x^{-1}$ is operator increasing. Applying the inequality in (38), we get

$$
M_{\ln x}(\bar{A}) \leq M_{x}(\bar{A}) .
$$

The double inequality in (41) follows by connecting the inequalities in (42) and (43).

Quasi-arithmetic operator means without applying operator convexity were also investigated in $[4,10]$.

\section{Conflict of Interests}

The author declares that there is no conflict of interests regarding the publication of this paper.

\section{References}

[1] C. Davis, "A Schwarz inequality for convex operator functions," Proceedings of the American Mathematical Society, vol. 8, pp. 4244, 1957.

[2] M. D. Choi, "A Schwarz inequality for positive linear maps on $\mathrm{C}^{*}$-algebras," Illinois Journal of Mathematics, vol. 18, pp. 565$574,1974$.

[3] F. Hansen, J. Pečarić, and I. Perić, "Jensen's operator inequality and its converses," Mathematica Scandinavica, vol. 100, no. 1, pp. 61-73, 2007.

[4] J. Mićić, Z. Pavić, and J. Pečarić, "Jensen's inequality for operators without operator convexity," Linear Algebra and its Applications, vol. 434, no. 5, pp. 1228-1237, 2011.

[5] Z. Pavić, "The applications of functional variants of Jensen's inequality," Journal of Function Spaces and Applications, vol. 2013, Article ID 194830, 5 pages, 2013.

[6] A. M. Mercer, "A variant of Jensen's inequality," Journal of Inequalities in Pure and Applied Mathematics, vol. 4, article 73, 2 pages, 2003.

[7] T. Furuta, J. Mićić Hot, J. Pečarić, and Y. Seo, Mond-Pečarić Method in Operator Inequalities, vol. 1, Element, Zagreb, Croatia, 2005.

[8] A. Matković, J. Pečarić, and I. Perić, "Refinements of Jensen's inequality of Mercer's type for operator convex functions," Mathematical Inequalities \& Applications, vol. 11, no. 1, pp. 113126, 2008.

[9] J. E. Pečarić, F. Proschan, and Y. L. Tong, Convex Functions, Partial Orderings, and Statistical Applications, vol. 187, Academic Press, Boston, Mass, USA, 1992.

[10] J. Mićić, Z. Pavić, and J. Pečarić, "The inequalities for quasiarithmetic means," Abstract and Applied Analysis, vol. 2012, Article ID 203145, 25 pages, 2012. 


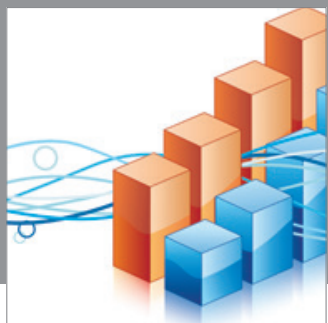

Advances in

Operations Research

mansans

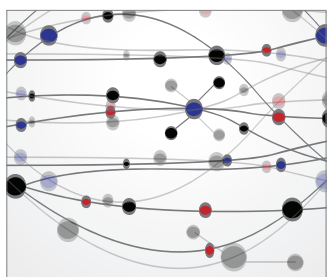

The Scientific World Journal
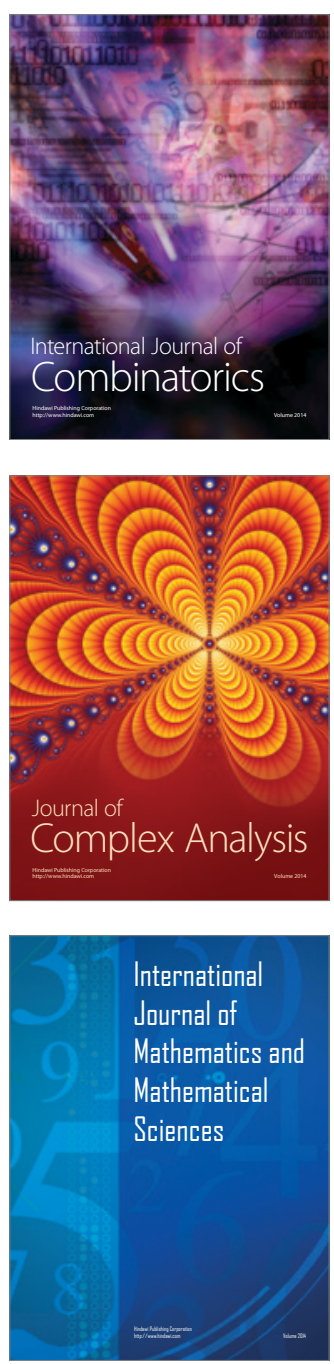
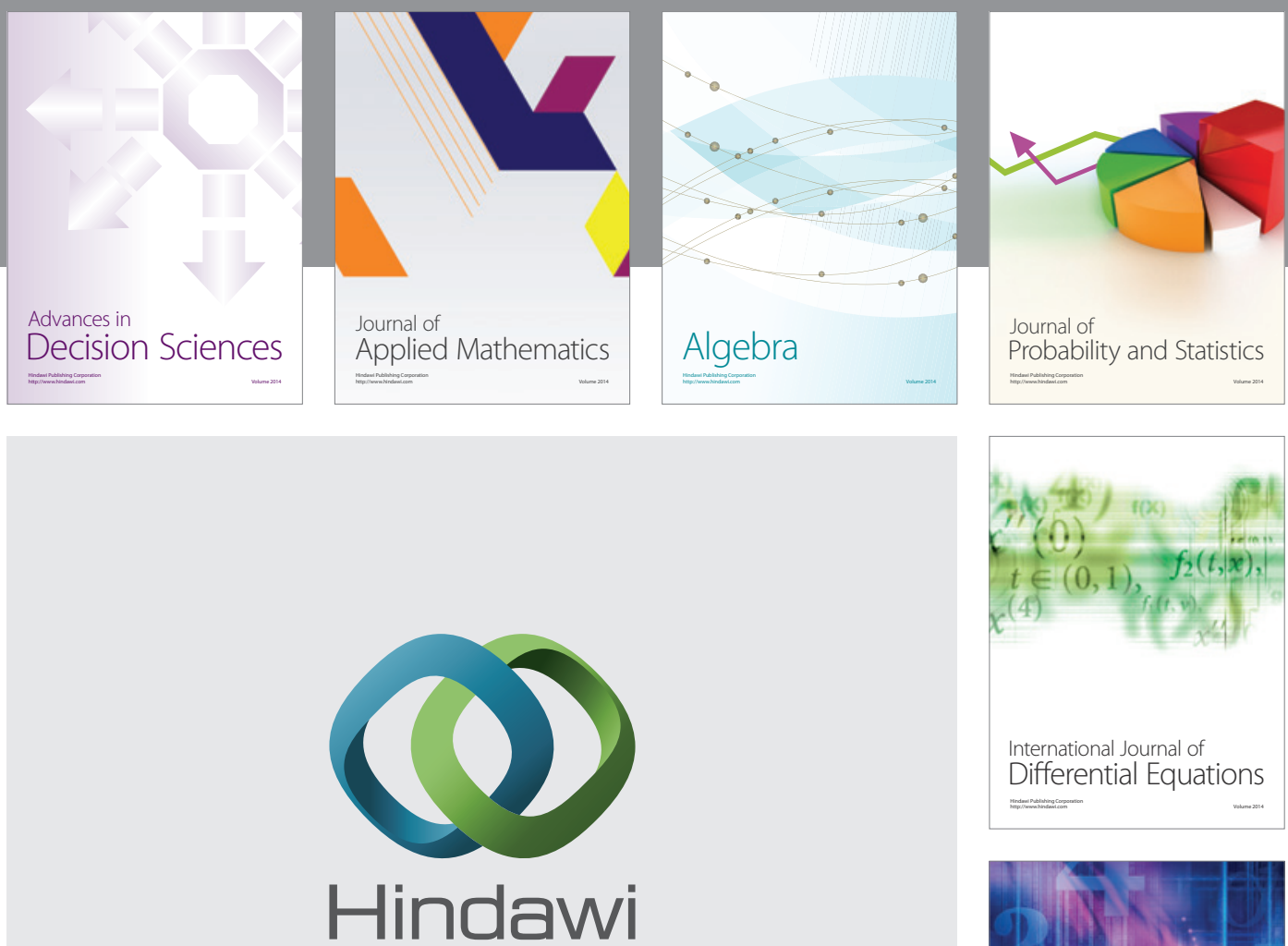

Submit your manuscripts at http://www.hindawi.com
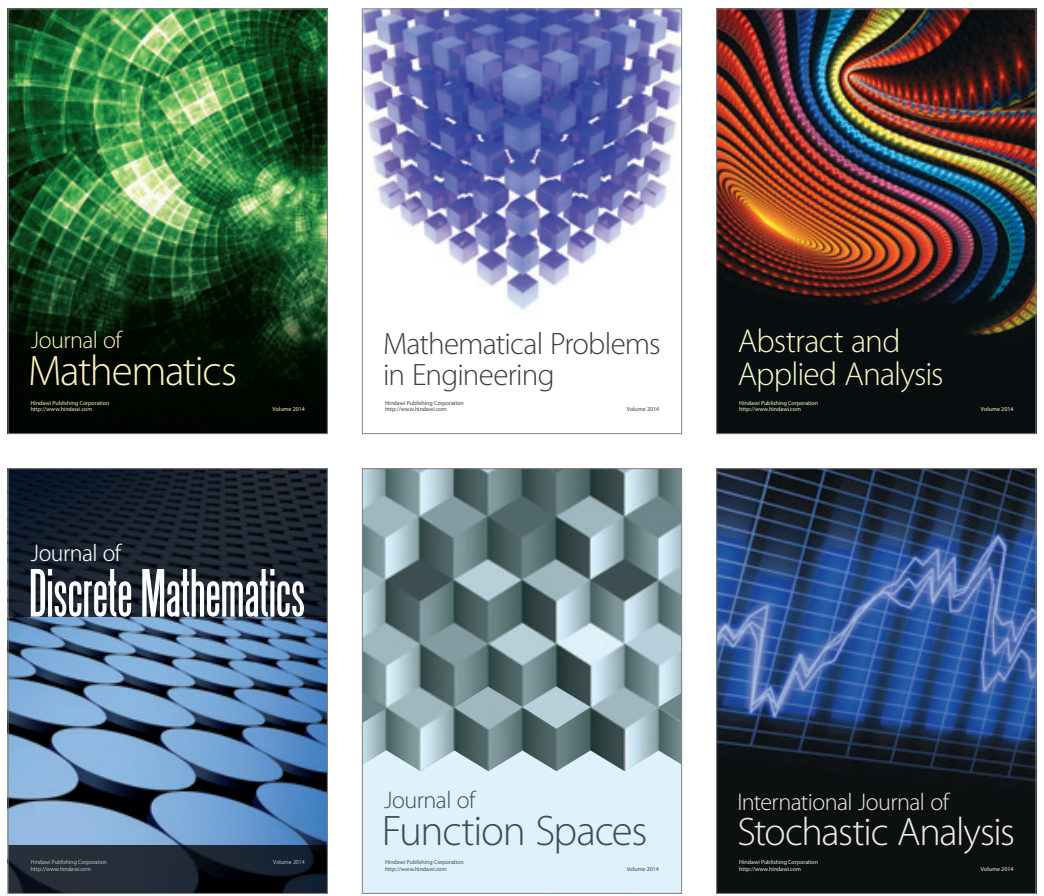

Journal of

Function Spaces

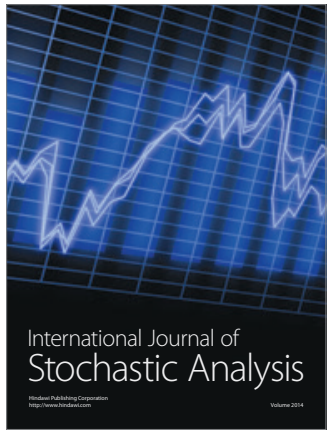

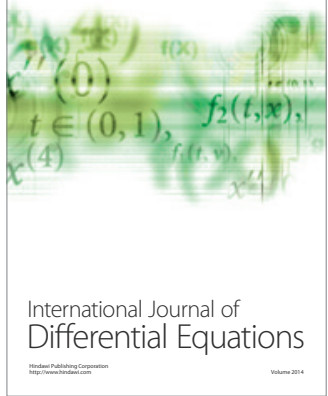
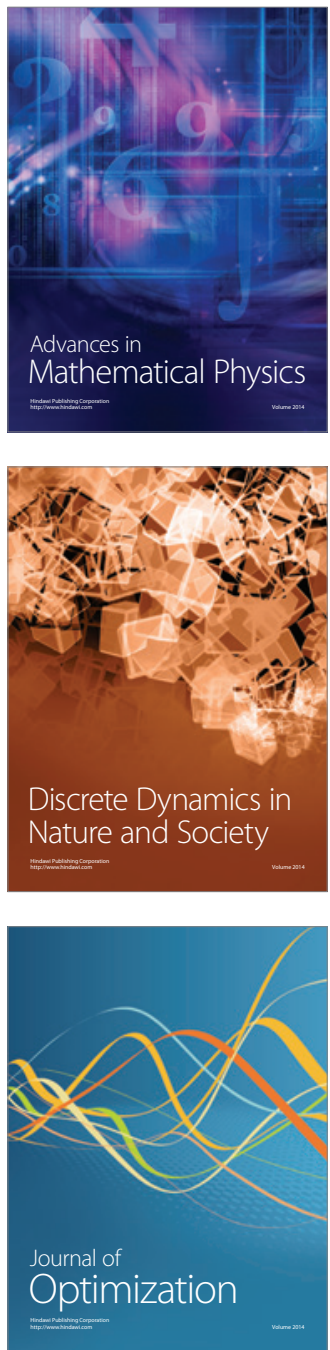